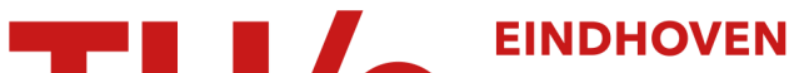 \\ UNIVERSITY OF \\ TECHNOLOGY
}

\section{Directional notch filter for motion control of flexible structures}

Citation for published version (APA):

Hoogendijk, R., Heertjes, M. F., Molengraft, van de, M. J. G., \& Steinbuch, M. (2014). Directional notch filter for motion control of flexible structures. Mechatronics, 24(6), 632-639.

https://doi.org/10.1016/j.mechatronics.2014.01.011

DOI:

10.1016/j.mechatronics.2014.01.011

Document status and date:

Published: 01/01/2014

\section{Document Version:}

Publisher's PDF, also known as Version of Record (includes final page, issue and volume numbers)

\section{Please check the document version of this publication:}

- A submitted manuscript is the version of the article upon submission and before peer-review. There can be important differences between the submitted version and the official published version of record. People interested in the research are advised to contact the author for the final version of the publication, or visit the $\mathrm{DOI}$ to the publisher's website.

- The final author version and the galley proof are versions of the publication after peer review.

- The final published version features the final layout of the paper including the volume, issue and page numbers.

Link to publication

\section{General rights}

Copyright and moral rights for the publications made accessible in the public portal are retained by the authors and/or other copyright owners and it is a condition of accessing publications that users recognise and abide by the legal requirements associated with these rights.

- Users may download and print one copy of any publication from the public portal for the purpose of private study or research.

- You may not further distribute the material or use it for any profit-making activity or commercial gain

- You may freely distribute the URL identifying the publication in the public portal.

If the publication is distributed under the terms of Article 25fa of the Dutch Copyright Act, indicated by the "Taverne" license above, please follow below link for the End User Agreement:

www.tue.nl/taverne

Take down policy

If you believe that this document breaches copyright please contact us at:

openaccess@tue.nl

providing details and we will investigate your claim. 


\title{
Directional notch filters for motion control of flexible structures
}

\author{
R. Hoogendijk*, M.F. Heertjes, M.J.G. van de Molengraft, M. Steinbuch \\ Department of Mechanical Engineering, Eindhoven University of Technology, Den Dolech 2, 5600 MB Eindhoven, The Netherlands
}

\section{A R T I C L E I N F O}

\section{Article history:}

Received 12 July 2013

Revised 29 December 2013

Accepted 13 January 2014

Available online 21 February 2014

\section{Keywords:}

Notch filtering

MIMO motion systems

Modal control

Loop-shaping techniques

\begin{abstract}
A B S T R A C T
A new method to design notch filters for MIMO motion control systems with flexible mechanical structures is proposed. The method involves so-called directional notch filters that work only in the direction of the targeted resonant mode. As a result, only one SISO notch filter is required per mode to suppress a resonance throughout the MIMO system. Compared to the conventional approach where a notch filter is placed and tuned in each of the separate control loops, the new approach reduces the order of the controller significantly and facilitates the design process. The directional notch filter is computed using either the input or output mode shapes of the system. A new numerical optimization method to obtain these mode shapes from frequency response data is described. Experiments on a flexible beam setup demonstrate the feasibility of the proposed method in practice.
\end{abstract}

(c) 2014 Elsevier Ltd. All rights reserved.

\section{Introduction}

Dealing with flexible dynamics of high-precision motion systems has become increasingly important due to increasing performance requirements for these systems. A clear example is given by wafer scanners in the semi-conductor industry. To achieve high accelerations in these scanners, the trend is toward lightweight mechanical designs. However, in general this results in a decrease in stiffness of these systems and causes the resonances of these systems to shift towards lower frequencies, thereby potentially affecting the stability of the system. This complex interaction between the mechanical design, the dynamics of the system, the actuation and sensor systems and the control design poses a challenging problem.

In industrial practice, high-precision multi-input-multi-output (MIMO) motion systems are generally controlled in a decentralized way $[1,2]$. To this end, MIMO motion systems are decomposed in single-input-single-output (SISO) systems by pre- and postmultiplying the plant with static decoupling matrices. As accurate frequency response data can be obtained at low costs for such systems, loop-shaping is used to design the SISO controllers [3]. The controllers usually contain notch filters to suppress high-frequency resonances that cause instability of the closed-loop system. However, at high frequencies the system is usually not decoupled. The reason for this is the interaction caused by the flexible dynamics of these systems. This means that the notch filters that

\footnotetext{
* Corresponding author. Tel.: +31402472796.

E-mail address: hoogendijkrob@gmail.com (R. Hoogendijk).
}

are designed in each SISO loop separately to suppress the resonances that appear in that loop, do not guarantee that the complete MIMO system is stable. This is because the system is not decoupled at these frequencies.

Ideally, it is possible to decouple all modes and address each of them in a separate control loop by modal decoupling, as described in [4], and applied by, e.g., [5-7]. Modal decoupling exploits the mode shapes to compute static transformation matrices to decouple the modes. However, the number of modes that can be decoupled is limited by the number of actuators and sensors. Flexible structures in principle have infinitely many modes such that perfect decoupling of all modes would require an infinite number of actuators and sensors.

The step toward dynamic decoupling matrices has been made to overcome this limitation, but with limited success only $[8,9]$. Using observers to estimate the modal states has also been studied extensively. In this field, independent modal space control [10] is the best known example. However, it is well known that these observers suffer from control and observation spillover of unmodelled modes, see [11]. Despite this disadvantage, independent modal space control remains a topic of active research, especially in those cases where a sufficient number of sensors is available, see [12-14] and the references therein.

Another disadvantage of dynamic decoupling and observer based strategies is that they often require a MIMO model of the system to be controlled. Accurate MIMO models for high-precision motion systems are not readily available, see [15,2].

In this paper, an alternative method to deal with the resonances in flexible MIMO motion systems is described. The dynamics of a flexible structure can be described by its modal representation, 
see $[16,17]$. In this representation, it is assumed that the transfer function of the system can be written as a summation of modes, each with a specific frequency, damping, and mode shape. These mode shapes give the modes a strong directionality in the MIMO system. Since each mode has a specific input and output direction, the control of these modes should take this directionality into account.

Directionality is the main difference between SISO and MIMO systems [18]. While it is often recognized that directions in MIMO systems are important, there are not many methods to design controllers that cope with this directionality, without resorting to full model-based control design methods. [19] describes a method to design a controller that counteracts disturbances in the appropriate directions. Contrary to [19], that is focussed on disturbance rejection, our method aims at using the directions of the flexible modes in the control design.

The directionality of the modes is utilized to design notch filters that have the correct directionality within the MIMO system. For this purpose, a SISO notch filter is distributed over the channels of the MIMO system according to the mode shape of the targeted resonant mode, creating a so-called directional notch filter. Conventionally, for each mode one notch filter per SISO loop is required to suppress the resonance in all channels of the system. A directional notch filter requires only one SISO notch filter such that the order of the controller is reduced and the design process is facilitated. Note that contrary to the available feedback, e.g., [20,21] and feedforward methods, e.g., [22] that rely on the repetitive nature of the motion task, our approach is completely independent of the setpoint. The proposed approach aims at enhancement of the dynamics of the system rather than the suppression of (repetitive) disturbances.

In the conventional way of designing notch filters, i.e., independent design of a controller for each SISO loop, merely the stability of the SISO loops is considered. After the loop shaping of the SISO loops, the stability of the MIMO system should be checked in view of the interaction between the loops. If the MIMO stability analysis shows that the system is unstable, it is in general not clear which SISO loop should be adjusted to stabilize the system. Adaptive notch filters as described in [23], are used for the automatic tuning of SISO notch filters. However, the multivariable nature of these systems is not taken into account. Another approach that is often used is sequential loop closing, see [24,25]. Although this technique does consider the interaction between the loops, the order in which the loops are closed is arbitrary, and again it is not obvious which loop should be adjusted in case the performance of the system is unsatisfactory.

Design of the directional notch filters is done in view of MIMO stability, which guarantees the stability of the overall system. In this paper, the characteristic loci [26] are used to design the directional notch filters. Other MIMO stability analysis methods could be used as well.

The transformation matrices are computed from the mode shapes of the modes that need to be suppressed. The mode shapes may be obtained from a parametric model of the system to be controlled, if such a model is available. However, as mentioned before, an accurate parametric model is often not available. Therefore we will reside to a data-based method to obtain the mode shapes. Many methods are known in modal analysis literature, see [27] for an overview. However, these methods require that the number of inputs and outputs exceed the number of modes. Therefore, this paper also describes a new method to obtain the mode shapes from the frequency response data directly.

In addition, experiments have been conducted on a prototype flexible motion system to validate the proposed theory. Summarizing, the main contributions of this paper are:
- a new method to design notch filters for MIMO systems,

- an algorithm to compute mode shapes from frequency response data,

- validation of the method by experiments.

This paper is organized as follows. First, the necessary concepts of the modal description are discussed in Section 2. Next, conventional notch filter design is discussed in Section 3. Section 4 explains the concept of directional notch filtering and elaborates on the computation of directional notch filters. Experiments are presented in Section 5, followed by conclusions in Section 6 .

\section{Modal description and notation}

Flexible structures are characterized by internal deformations of a structure. The dynamics of these internal deformations can be described in nodal or modal coordinates. Nodal coordinates typically represent the position and velocity of each node in the structure. A large number of nodes is required to obtain an accurate description of the system, which implies that accurate nodal models are generally of high order. To limit the model order, the dynamics of flexible structures are often described using the modal representation, see e.g., [17] or [16]. In this description, the deformation of a flexible structure is described in terms of a limited number of modes and mode shapes. These modes are mutually independent (assuming proportional damping), contrary to the nodal coordinates, which simplifies the analysis. Several modal forms are possible, see [16]. In this paper a modal description with state vector

$\mathbf{x}=\left[\begin{array}{c}\mathbf{q}_{m} \\ \dot{\mathbf{q}}_{m}\end{array}\right]$,

is chosen, where $\mathbf{q}_{m}$ represent the modal displacements and $\dot{\mathbf{q}}_{m}$ the modal velocities. Both $\mathbf{q}_{m}$ and $\dot{\mathbf{q}}_{m}$ are vectors of length $n$, where $n$ is the number of modes in the model. The $2 n$ equations of motion are given by

$\dot{\mathbf{x}}=\mathbf{A x}+\mathbf{B u}$

$\mathbf{y}=\mathbf{C x}+\mathbf{D u}$

with

$\mathbf{A}=\left[\begin{array}{cc}\mathbf{0} & \mathbf{I}_{n} \\ \mathbf{\Omega}^{2} & -2 \mathbf{Z} \mathbf{\Omega}^{2}\end{array}\right], \quad \mathbf{B}=\left[\begin{array}{c}\mathbf{0} \\ \mathbf{B}_{m}\end{array}\right]$,

$\mathbf{C}=\left[\begin{array}{ll}\mathbf{C}_{m} & \mathbf{0}\end{array}\right], \quad \mathbf{D}=\mathbf{0}$,

where

$\boldsymbol{\Omega}=\operatorname{diag}\left(\omega_{1}, \ldots, \omega_{n}\right)$,

is the matrix that contains the eigenfrequencies $\omega_{i}$ of the modes and where

$\mathbf{Z}=\operatorname{diag}\left(\zeta_{1}, \ldots, \zeta_{n}\right)$,

is the matrix that contains the modal damping ratios $\zeta_{i}$ of the modes. Consequently, the A matrix is of dimension $2 n \times 2 n$. B is $2 n \times n_{i}$, with $n_{i}$ the number of actuators. Since only force actuators are considered, the first $n$ rows of $\mathbf{B}$ are zero. The lower, non-zero part of $\mathbf{B}$ is denoted with $\mathbf{B}_{m}$ and is given by

$\mathbf{B}_{m}=\left[\begin{array}{c}\mathbf{b}_{m 1}^{T} \\ \mathbf{b}_{m 2}^{T} \\ \vdots \\ \mathbf{b}_{m n}^{T}\end{array}\right]$. 
Each row $\mathbf{b}_{m i}^{T}$ contains the mode shape of mode $i \in\{1,2, \ldots, n\}$ as being controlled at the $n_{i}$ actuator locations, i.e., the input mode shape of mode $i$. $\mathbf{C}$ has dimensions $n_{0} \times 2 n$, with $n_{o}$ the number of sensors. The last $n$ columns of $\mathbf{C}$ are equal to zero since only position sensors are considered. The first $n$ non-zero columns are denoted with $\mathbf{C}_{m}$ and are given by

$\mathbf{C}_{m}=\left[\begin{array}{llll}\mathbf{c}_{m 1} & \mathbf{c}_{m 2} & \cdots & \mathbf{c}_{m n}\end{array}\right]$.

Each column $\mathbf{c}_{m i}$ contains the mode shape of mode $i$ as observed at the $n_{o}$ sensor locations. $\mathbf{c}_{m i}$ will further be referred to as the output mode shape of mode $i$.

The state space system (2) contains $n$ independent second-order differential equations, one for each mode. This can be seen by substitution of (3) in (2), which gives

$\mathbf{y}(s)=\mathbf{H}(s) \mathbf{u}(s)$,

where

$$
\begin{aligned}
\mathbf{H}(s) & =\mathbf{C}\left(s \mathbf{I}_{2 n}-\mathbf{A}\right)^{-1} \mathbf{B}=\mathbf{C}_{m}\left(\mathbf{\Omega}^{2}+s^{2} \mathbf{I}_{n}+2 s \mathbf{Z} \boldsymbol{\Omega}\right)^{-1} \mathbf{B}_{m} \\
& =\sum_{i=1}^{n} \frac{\mathbf{C}_{m i} \mathbf{b}_{m i}^{T}}{s^{2}+2 s \zeta i \omega_{i}+\omega_{i}^{2}},
\end{aligned}
$$

where the special structure of the $\mathbf{B}$ and $\mathbf{C}$ matrices and the fact that $\mathbf{\Omega}$ and $\mathbf{Z}$ are diagonal is used. Eq. (9) shows that the modes are independent, the transfer from inputs $\mathbf{u}$ to outputs $\mathbf{y}$ is a summation of individual modal contributions. Furthermore, it can be observed from (9) that each mode has a very specific directionality in the MIMO system, which is completely determined by the numerator terms in (9). For each mode, the denominator is a scalar valued function. This means that the directionality is determined by the constant matrix $\mathbf{c}_{m i} \mathbf{b}_{m i}^{T}$ in the numerator. Since this matrix is built up from a multiplication of a column vector with a row vector, the rank of the matrix is one. This shows that each mode has a very specific directionality, determined by its input and output mode shape. This directionality will be utilized in the method proposed in the remainder of this paper.

\section{Conventional notch filters}

Multi-input-multi-output (MIMO) motion systems are often controlled in a decentralized way. Conventionally, static decoupling matrices $\mathbf{T}_{y}$ and $\mathbf{T}_{u}$ are used to transform the inputs and outputs of the system $\mathbf{H}(s)$ to a new set of coordinates according to

$\mathbf{H}_{d}(s)=\mathbf{T}_{y} \mathbf{H}(s) \mathbf{T}_{u}$,

where $\mathbf{H}_{d}(s)$ represents the decoupled system. There are several advantages to this approach. Firstly, the transformation matrices are chosen in such a way that they minimize the coupling between the subsequent channels, which makes it possible to apply singleinput-single-output (SISO) control design techniques to control a MIMO system. This simplifies the control design significantly. Secondly, the new coordinates can be chosen in such a way that they are aligned with the performance variables. This simplifies the setpoint design and performance assessment.

Motion systems are often decoupled in their rigid body modes, since these modes enable the system to perform the motion tasks for which it is designed. For example, a 6 degrees-of-freedom (DOF) motion system will have six rigid body modes for which $\omega_{i}=0$ for $i \leqslant 6$, see (9). The system will be decoupled in these modes such that a controller can be designed for each DOF separately. However, every motion system has a finite stiffness, which means that internal deformations will occur during the motion task due to the non-rigid body, or flexible, modes of the system. In the example of the 6-DOF motion system this means that in (9) $\omega_{i} \neq 0$ for $i>6$.
Lightweight motion systems are often equipped with more sensors and actuators than rigid-body modes, to increase control authority over the internal deformations of the system. This is sometimes referred to as overactuation and gives more freedom in the selection of the decoupling matrices. Moreover, it enables modal decoupling where besides the rigid body modes a limited number of non-rigid body modes are decoupled as well. However, the number of modes that can be controlled explicitly is limited by the number of actuators and sensors in the system. A flexible structure is an infinite dimensional system having infinitely many modes such that in principle it is not possible to decouple all modes.

The remaining modes, which are not decoupled, will be visible to some extent in the transfer functions contained in $\mathbf{H}_{d}(s)$ depending on their mode shapes. The modes that affect closed-loop stability are suppressed using loop-shaping notch filters in the SISO loops. This, however, poses the following problem. Firstly, there is no guarantee that the MIMO system is stable when all SISO loops are designed to be stable, since the system is generally not decoupled at the frequencies of the resonances. Therefore, MIMO stability has to be checked afterwards. Secondly, should the MIMO system no longer satisfy the required stability properties, it is no straightforward task to determine in which loop a notch should be adjusted as to ensure MIMO stability altogether. Therefore, a different approach is needed.

\section{Directional notch filters}

The directionality of the modes can be utilized to design notch filters that have the correct directionality in the MIMO system. We call this directional notch filtering. The idea is depicted in Fig. 1 for mode $i$. The MIMO system $\mathbf{H}(s)$ is controlled in a decentralized way by diagonal controller $\mathbf{C}(s)$, where the decoupling is achieved by the input decoupling matrix $\mathbf{T}_{u}$ and output decoupling matrix $\mathbf{T}_{y}$. To this standard decentralized control loop, a directional notch is added at the plant input to target mode $i$ of the system. The directional notch filter consists of two static transformation matrices $\mathbf{T}_{i n, i}$ and $\mathbf{T}_{i n, i}^{-1}$ and a matrix $\mathbf{N}_{i}(s)$ that contains a notch filter at one of its entries.

\subsection{Directional notch filtering concept}

The transformation matrix $\mathbf{T}_{i n, i}$ in Fig. 1 transforms the input directions of the plant such that the input direction of mode $i$ is isolated from the other input directions. This is achieved by the $n_{i} \times n_{i}$ matrix

$$
\mathbf{T}_{i n, i}=\left[\begin{array}{ll}
\mathbf{b}_{m i} & \operatorname{Ker}\left(\mathbf{b}_{m i}^{T}\right)
\end{array}\right]
$$

which consists of the input mode shape $\mathbf{b}_{m i}$ of mode $i$ and its kernel. The kernel of $\mathbf{b}_{m i}^{T}$ contains the $n_{i}-1$ vectors that are perpendicular to the input mode shape of mode $i$. In this way, $\mathbf{T}_{i n, i}$ always has full rank, such that the inverse transformation matrix $\mathbf{T}_{i n, i}^{-1}$ exists. The transformation matrix $\mathbf{T}_{i n, i}$ combined with the input matrix $\mathbf{B}_{m}$ of (3), yields

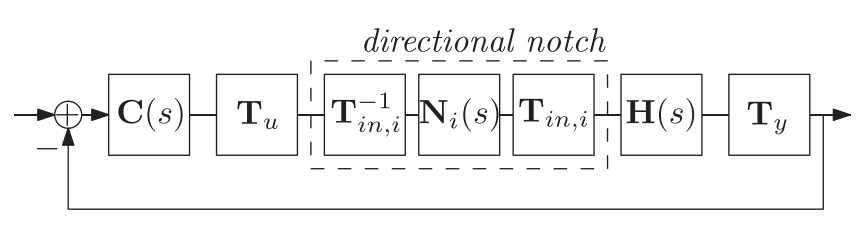

Fig. 1. Feedback loop with directional notch filter. 


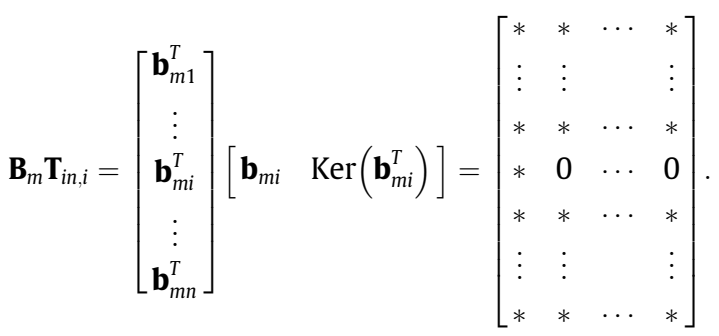

Since the $i^{\text {th }}$ row is zero, except for the first element, mode $i$ can only be transferred through the first channel of the transfer function $\mathbf{H}(s) \mathbf{T}_{i n, i}$. Thus, the transformation makes the $i^{\text {th }}$ mode uncontrollable for all inputs except for the first input. In this way, the resonance associated with this mode can be suppressed with one SISO notch filter in the first input channel. The diagonal notch filter for the transformed system is therefore given by

$\mathbf{N}_{i}(s)=\left[\begin{array}{cc}N_{i}(s) & \mathbf{0} \\ \mathbf{0} & \mathbf{I}_{n_{i}-1}\end{array}\right]$,

where

$N_{i}(s)=\frac{s^{2}+2 s \zeta_{N i, n} \omega_{N i, n}+\omega_{N i, n}^{2}}{s^{2}+2 s \zeta_{N i, d} \omega_{N i, d}+\omega_{N i, d}^{2}}$

with $\zeta_{N i, n}, \zeta_{N i, d}$ and $\omega_{N i, n}, \omega_{N i, d}$ the damping ratios and frequencies of the notch filter that are tuned for mode $i$ as will be described in the next section. The other channels are unchanged, hence the identity matrix in (13). In order to control the system in the original directions, the inverse of the transformation matrix, i.e., $\mathbf{T}_{i n, i}^{-1}$, is added after notch filtering. The open-loop $\mathbf{L}(s)$ with the directional notch filter is thus given by

$$
\mathbf{L}(s)=\mathbf{T}_{y} \mathbf{H}(s) \mathbf{T}_{i n, i} \mathbf{N}_{i}(s) \mathbf{T}_{i n, i}^{-1} \mathbf{T}_{u} \mathbf{C}(s) .
$$

The directional notch performs a local adjustment of the frequency response of the system. Just as a conventional notch, it is local in frequency because the notch is only affecting a narrow frequency band. However, the directional notch is also local in terms of direction; it only acts in the direction of the mode shape of the targeted resonance. Note that the approach can be applied to multiple modes by adding multiple directional notches in series. In that case the open-loop becomes

$$
\mathbf{L}(s)=\mathbf{T}_{y} \mathbf{H}(s) \prod_{i}\left(\mathbf{T}_{i n, i} \mathbf{N}_{i}(s) \mathbf{T}_{i n, i}^{-1}\right) \mathbf{T}_{u} \mathbf{C}(s),
$$

where $i$ runs over all targeted modes.

Motion systems often require a non-constant decoupling, for example when the performance location of the system changes over time. In that case the decoupling will also be required to change over time, i.e., to become position dependent. As a result, conventional notch filters either have to be chosen robustly, at the cost of performance, or different notch filters have to be used at different points of interest on the structure, which comes at the cost of controller complexity. Since the directional notch is placed directly before the plant, it is independent of the decoupling of the system. This means that it is neither necessary to design the notches very robustly, nor to reside to different notches at different performance locations. Directional notch filtering can be seen as an enhancement of the plant, independent of the controllers for the decoupled degrees of freedom. The plant with directional notch filters can be controlled at a higher bandwidth compared to the plant without directional notch filters. However, the achievable performance of the overall system still depends on the limitations of this enhanced system. These limitations are the quality of the decoupling, fundamental performance limits such as right-half-plane zeros, available phase lead, to name but a few.
In the previous analysis, the directional notches were applied to the inputs of the plant. They can also be applied to the output of the plant, however. In that case the $n_{0} \times n_{0}$ transformation matrix

$\mathbf{T}_{\text {out }, i}=\left[\begin{array}{ll}\mathbf{c}_{m i} & \operatorname{Ker}\left(\mathbf{c}_{m i}^{T}\right)\end{array}\right]^{T}$,

has to be used, which is determined by the output mode shape $\mathbf{c}_{m i}$ of mode $i$. In terms of the outputs of (3), the transformation at the output combines the output matrix $\mathbf{C}_{m}$, giving

$$
\begin{aligned}
\mathbf{T}_{\text {out }, i} \mathbf{C}_{m}= & {\left[\begin{array}{c}
\mathbf{c}_{m i}^{T} \\
\operatorname{Ker}\left(\mathbf{c}_{m i}^{T}\right)^{T}
\end{array}\right]\left[\begin{array}{llllll}
\mathbf{c}_{m 1} & \cdots & \mathbf{c}_{m i} & \cdots & \mathbf{c}_{m n}
\end{array}\right] } \\
= & {\left[\begin{array}{ccccccc}
* & \cdots & * & * & * & \cdots & * \\
* & \cdots & * & 0 & * & \cdots & * \\
\vdots & & \vdots & \vdots & \vdots & & \vdots \\
* & \cdots & * & 0 & * & \cdots & *
\end{array}\right] }
\end{aligned}
$$

That is, the $i^{\text {th }}$ mode becomes unobservable in all output channels except for the first one. Again it is possible to suppress this resonance with a single SISO notch in the first output channel. The open-loop with directional notch filter for mode $i$ at the output is defined as

$\mathbf{L}(s)=\mathbf{T}_{y} \mathbf{T}_{\text {out }, i}^{-1} \mathbf{N}_{i}(s) \mathbf{T}_{\text {out }, i} \mathbf{H}(s) \mathbf{T}_{u} \mathbf{C}(s)$.

Addressing multiple modes is done by adding extra directional notch filters in series to obtain

$\mathbf{L}(s)=\mathbf{T}_{y} \prod_{i}\left(\mathbf{T}_{\text {out }, i}^{-1} \mathbf{N}_{i}(s) \mathbf{T}_{\text {out }, i}\right) \mathbf{H}(s) \mathbf{T}_{u} \mathbf{C}(s)$.

\subsection{Design of the directional notch filter}

As discussed in Section 3, the conventional loop-shaping approach is to place SISO notch filters in each decentralized SISO loop. However, the non-decoupled modes will appear in all transfer functions of the MIMO system, so several SISO loops will contain notch filters for the same resonant modes. This unnecessarily increases the order of the controller. Furthermore, the SISO design gives no guarantees regarding MIMO stability.

Design of the directional notch filter will be shown to be straightforward. The transformation matrices $\mathbf{T}_{\text {in.i }}$ from (11) (or $\mathbf{T}_{\text {out }, i}$ from (17) in the case that the directional notch is used at the plant output) ensures that the directional notch filter works in the right direction of the MIMO system. Not only is the order of the controller reduced in this way, but it also facilitates the design process: per mode only a single SISO notch filter (14) has to be tuned. This can be done in combination with a MIMO stability analysis method such as the characteristic loci or the MIMO Nyquist plot. The characteristic loci will be used in this paper. The overall control design procedure becomes as follows:

1. decouple the system with static decoupling matrices $\mathbf{T}_{u}$ and $\mathbf{T}_{y}$,

2. design SISO controllers for the decoupled system $\mathbf{H}_{d}(s)$ disregarding the high-frequency resonances,

3. draw the characteristic loci and assess which resonances need notch filters,

4. compute directional notch filters for these resonances,

5. design the SISO notch filters $N_{i}(s)$ using characteristic loci.

In this paper, the need for notch filters (step 3 ) is determined on the basis of the characteristic loci plot. The SISO notch filters $N_{i}(s)$ are tuned (step 5) such that the characteristic loci lie outside a circle with radius 0.5 around the -1 point, thus ensuring a design with sufficient robustness margins. Of course, other stability measures, design methods, and/or robustness criteria can be chosen. 


\subsection{Obtaining the mode shapes}

One aspect that has not been discussed so far is how to obtain the mode shapes that are used to construct the $\mathbf{T}_{i n, i}$ or $\mathbf{T}_{\text {out }, i}$ matrices. One approach is to derive a modal model using Finite Element Modeling (FEM). For motion control purposes, however, FEM models are either expensive or not accurate enough. Alternatively, (modal) identification techniques can be used, see [28] for a recent overview of this extensively studied field of research. The disadvantage of modal identification techniques is that they require that the number of sensors exceeds the number of modes, which is generally not the case in our field of application.

In this paper, an alternative, data-based approach is used to find the input or output mode shapes needed to construct $\mathbf{T}_{\text {in,i }}$ or $\mathbf{T}_{\text {out }, i}$. Since only the mode shape and not a complete modal model is required, a relatively straightforward approach can be used. The idea will be explained for the case that the directional notch is placed at the input side of the system. The derivation is similar for the case when the directional notch is placed at the output side of the system.

The idea is to find the input mode shape $\mathbf{b}_{m i}$. A way to do so is by inspecting whether the corresponding matrix $\mathbf{T}_{i n, i}$ causes mode $i$ to be affected by the first input only. The frequency response can be used to verify this. If $\mathbf{T}_{i n, i}$ is in the desired direction, the resonance peak of that mode will only appear in the first column of $\mathbf{H T}_{i n, i}$. For this purpose, the value of the transformed system at the resonance frequency $\omega_{i}$ of mode $i$ will be used. This is denoted with

$\mathbf{M}\left(j \omega_{i}\right)=\mathbf{H}\left(j \omega_{i}\right) \mathbf{T}_{i n, i}$,

while each of the entries of $\mathbf{M}\left(j \omega_{i}\right)$ with dimension $n_{0}$ times $n_{i}$ are denoted with $m_{k l}$. The cost function $J$ for this optimization can then be formulated as

$J=\min _{\mathbf{b}_{m i}} \sum_{k=1}^{n_{0}} \sum_{l=2}^{n_{i}}\left|m_{k l}\right|$.

The cost function $J$ represents a summation of all elements in $\mathbf{M}\left(j \omega_{i}\right)$, except for the first column. In this way the cost function is minimal if the resonance peak of mode $i$ is only visible in the first column of $\mathbf{H}(j \omega) \mathbf{T}_{i n, i}$.

Stating the problem of finding the mode shape in this way calls for numeric optimization techniques to find the minimum of (22). This requires a proper parametrization of $\mathbf{b}_{m i}$. Since only the direction and not the magnitude of $\mathbf{b}_{m i}$ is important, $\mathbf{b}_{m i}$ can parametrized using $n_{i}-1$ angles [8] giving

$$
\mathbf{b}_{m i}=\left[\begin{array}{c}
\prod_{m=1}^{n_{i}-1} \cos \left(\theta_{m}\right) \\
\sin \left(\theta_{n_{i}-1}\right) \prod_{m=1}^{n_{i}-2} \cos \left(\theta_{m}\right) \\
\sin \left(\theta_{n_{i}-2}\right) \prod_{m=1}^{n_{i}-3} \cos \left(\theta_{m}\right) \\
\vdots \\
\sin \left(\theta_{2}\right) \cos \left(\theta_{1}\right) \\
\sin \left(\theta_{1}\right)
\end{array}\right]
$$

which saves one optimization parameter, but more important, yields the optimization bounded as all angles range between $-\pi$ and $\pi$. With this parametrization, (22) represents a cheap optimization problem, since the number of parameters is only $n_{i}-1$. The cost function is also relatively simple and numerically not computationally demanding because it does not contain very large matrices. However, the optimization is non-linear and convexity properties are likely to be lost, which is why we reside to genetic algorithms to solve the optimization problem.

Whether the computation of the mode shapes is based on a parametric model of the system or on optimization routines, the stability analysis will always be performed completely data based. Computation of the characteristic loci or the MIMO Nyquist plot requires only frequency response data of the system. This means that errors in the approximation of the mode shapes, will be visible in the MIMO stability analysis.

\section{Results}

\subsection{Experiment setup}

To validate the approach, experiments have been conducted on a prototype lightweight motion system. The system, shown in Fig. 2, consists of a flexible beam with a collocated set of three actuators and three sensors. This beam setup approximates the dynamics such as found in flexible lightweight motion systems like lithographic machines. The dynamics of these systems are characterized by a combination of rigid body modes, which are the required degrees of freedom, and unwanted flexible modes that can affect the stability and limit performance of the closed-loop system. The beam can only move in $y$-direction and rotate about the $z$-axis, the other rigid body degrees of freedom are fixed. Internal deformations of the beam are mainly in the $y$-direction since this is the direction of the beam that has the lowest stiffness.

For the experiments in this paper, only the outer two actuators (A1, A3) and sensors (S1, S3) of the setup are used. Due to symmetry, all mode shapes are either symmetric or antisymmetric with respect to the centre of the beam. This means that decoupling toward the centre would decouple all flexible modes as well. As this is an exceptional situation, the decoupling is chosen toward a more challenging point between S1 and S2. Namely, the point where the coordinate system is drawn in Fig. 2. In this way, the non-rigid-body modes will be coupled in the (rigid-body) decoupled system. The decoupling matrices are given by

$\mathbf{T}_{y}=\left[\begin{array}{cc}1 & 1 \\ 3 & -1\end{array}\right], \quad \mathbf{T}_{u}=\mathbf{T}_{y}^{-1}$.

The frequency response of the decoupled system is shown in Fig. 3. The goal is to control the rigid body degrees of freedom with a bandwidth of $50 \mathrm{~Hz}$. From the relative gain array (RGA), plotted in Fig. 4, it can be observed that around the target bandwidth frequency, the decoupling is sufficiently good. The diagonal terms are around $0 \mathrm{~dB}$ at $50 \mathrm{~Hz}$, while the off-diagonal terms are much smaller $(-30 \mathrm{~dB})$. This favours decentralized control.

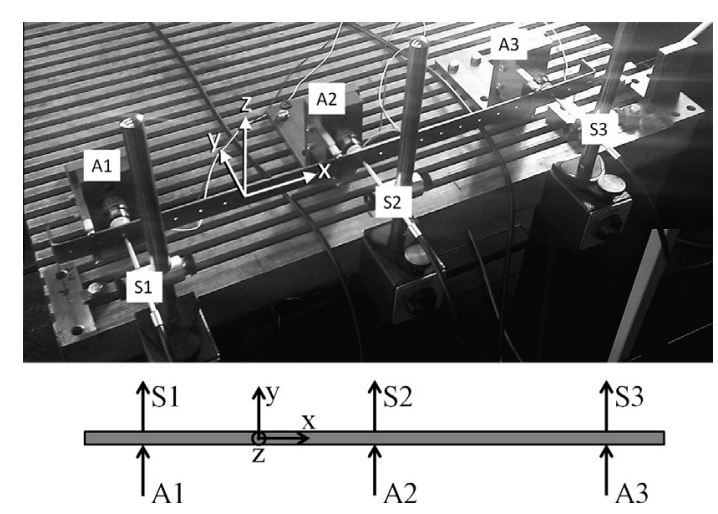

Fig. 2. Photo and schematic representation of the beam setup. The system has three actuators A1-A3 and sensors S1-S3. 


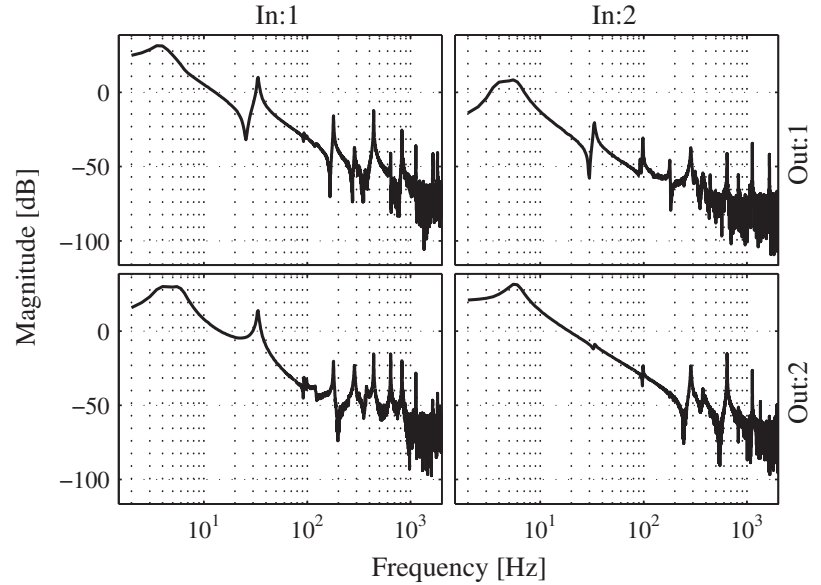

Fig. 3. Frequency response of the decoupled beam setup.

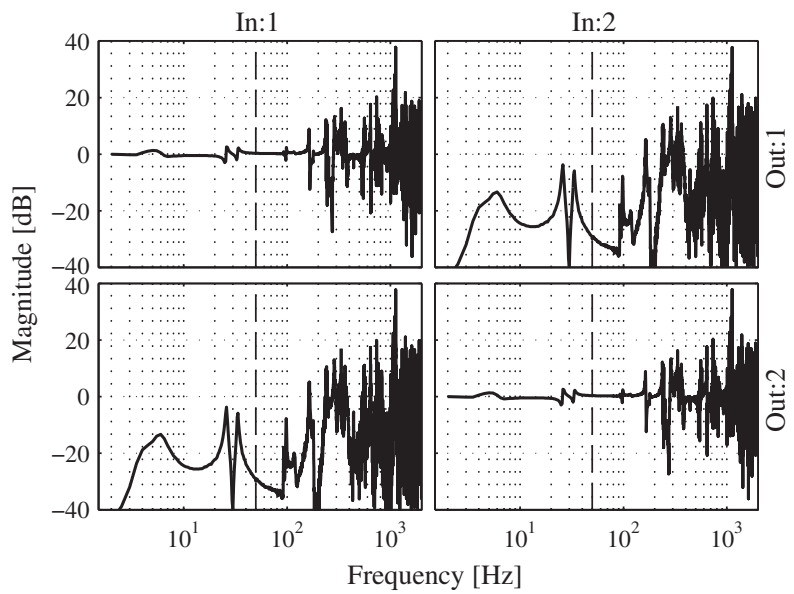

Fig. 4. Relative gain array. Decoupling is sufficiently obtained around the desired bandwidth $(50 \mathrm{~Hz})$, but lost at high frequencies.

\subsection{Decentralized control}

Two SISO controllers are designed using conventional loopshaping techniques on the two SISO open-loops, see Fig. 5. The controllers consist of a lead-filter, a low-pass filter, and a limited set of notch filters. The controller for the first loop has a notch filter at

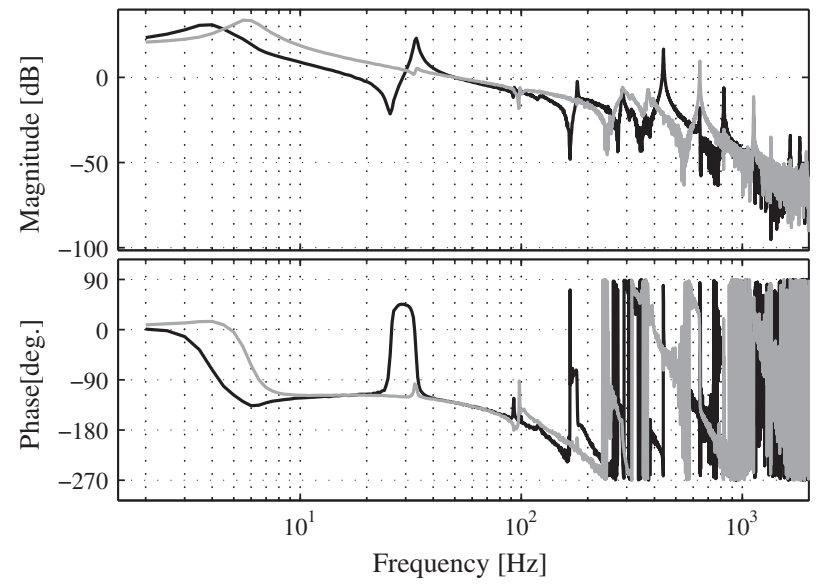

Fig. 5. Open loops of the first (black) and second (grey) loop for conventional decentralized control.
$179 \mathrm{~Hz}$, while the second loop has notch filters at $99 \mathrm{~Hz}$ and $286.5 \mathrm{~Hz}$. These notch filters are tuned such that the SISO Nyquist curves lie outside a circle with radius 0.5 around the Nyquist stability point at $(-1,0)$, see Fig. 6 . This guarantees that magnification of the corresponding sensitivity function $\leqslant 6 \mathrm{~dB}$ and the amplitude margins $\geqslant 6 \mathrm{~dB}$. However, Fig. 4 shows that the system is not decoupled the high frequencies. This means that a MIMO stability test is required to check stability of the MIMO system. For this purpose, the characteristic loci are plotted in Fig. 7.

The characteristic loci violate the previous circle constraint, showing that the stability margins are not as good as they appeared from SISO analysis. It can be concluded that the notch filter at $286.5 \mathrm{~Hz}$ is not applied in the proper direction of the MIMO system. This could be expected, since the system is not decoupled at this frequency. One solution might be to add a notch filter at $286.5 \mathrm{~Hz}$ in the first loop as well. However, this increases the order of the controller because it adds two states to the controller and it is not clear how to tune this notch filter.

\subsection{Directional notch filtering}

As conventional loop-shaping control design cannot deal properly with the coupled dynamics of the high-frequency resonances, the directional notch filtering concept is applied next. Firstly, the SISO controllers are designed disregarding high-frequency

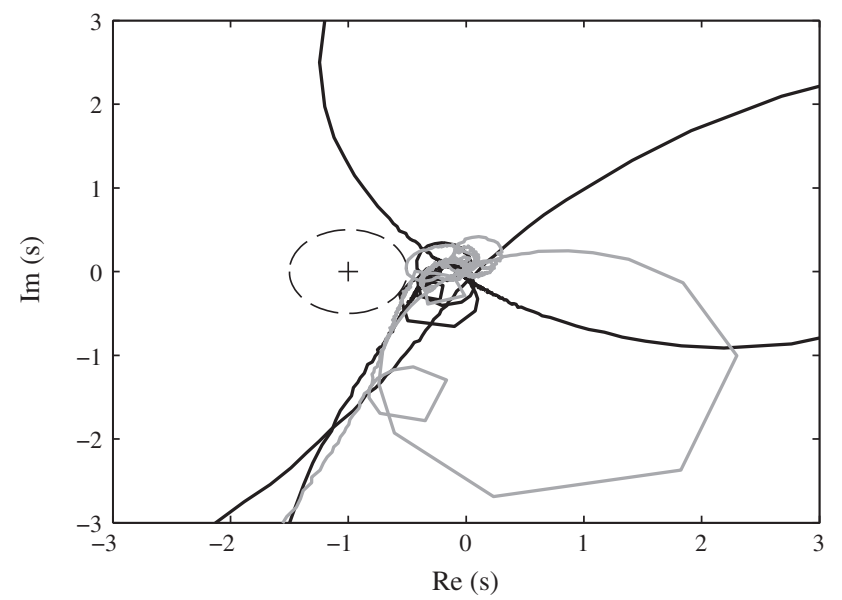

Fig. 6. Nyquist plots of the first (black) and second (grey) loop for conventional decentralized control.

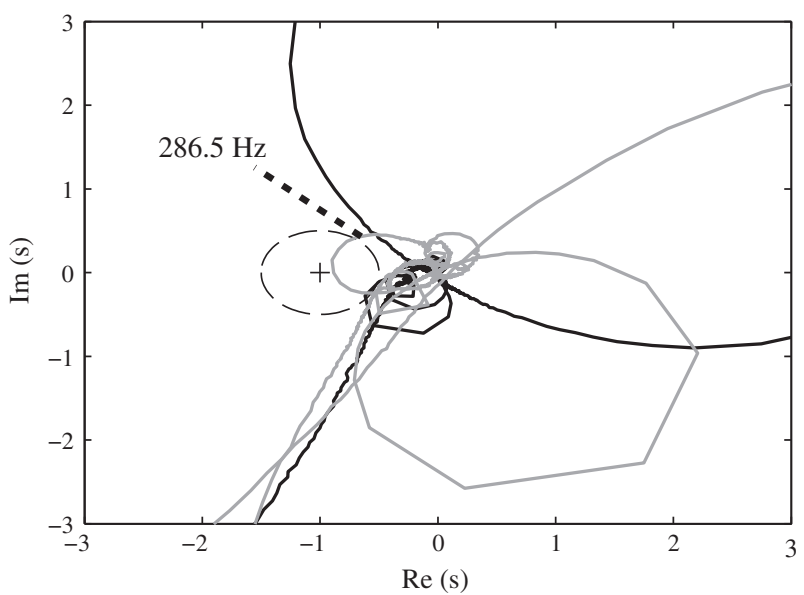

Fig. 7. Characteristic loci of the open-loop for conventional decentralized control. 
resonances. For the beam setup, these SISO controllers consist of the same lead lag and a low-pass filter as used in the conventional design approach, yet without the notch filters. The characteristic loci of the open-loop are depicted in Fig. 8. Three resonances at 99, 179 and $286.5 \mathrm{~Hz}$ lie within the circle around the Nyquist stability point. Directional notch filters, placed at the input of the plant, will be applied to these three modes. The modes will be numbered mode 1,2 and 3.

The input mode shapes $\mathbf{b}_{m 1}, \mathbf{b}_{m 2}$ and $\mathbf{b}_{m 3}$ of each mode are determined first, using the optimization method as described in Section 4.3. For this $2 \times 2$ system, the direction of each mode $i$ can be parametrized with one angle only. Thus in this case

$\mathbf{b}_{m i}=\left[\begin{array}{c}\cos \left(\theta_{i}\right) \\ \sin \left(\theta_{i}\right)\end{array}\right]$

where $i \in\{1,2,3\}$. The goal of the optimization is to find the value of $\theta_{i}$ for which the resonance peak of mode $i$ disappears from the second column of the frequency response plot of $\mathbf{H}(j \omega) \mathbf{T}_{i n, i}$. Three optimizations with a genetic algorithm are conducted with an initial population of 100 over 20 generations, which take less than a second to run on a normal pc. The result of the optimization is that

$\theta_{1}=-19.63^{\circ}$

$\theta_{2}=48.78^{\circ}$

$\theta_{3}=-45.56^{\circ}$.

These angles are used to compute the transformation matrices $\mathbf{T}_{i n, 1}, \mathbf{T}_{i n, 2}$ and $\mathbf{T}_{i n, 3}$ for each of the three directional notches that are used for this system. For each mode, it is verified that the resonance peak indeed disappears from the second column of $\mathbf{H}(j \omega) \mathbf{T}_{i n, i}$. Fig. 9 shows this for mode 2 . While the resonance at $298 \mathrm{~Hz}$ was visible in all transfers of the original system, the transformed system $\mathbf{H}(j \omega) \mathbf{T}_{i n, 2}$ no longer has the resonance in the second column of the frequency response matrix. This means that, at that point in the feedback loop, the system is decoupled with respect to mode 2 such that a single SISO notch filter in the first input channel is sufficient to remove this mode from the MIMO system as a whole.

Now that the direction of each mode is known, the next step is to tune the three SISO notch filters in the directional notch filters such that the characteristic loci from Fig. 8 lie outside the circle around the Nyquist stability point. Fig. 10 shows the characteristic loci of the system with the tuned directional notches. The MIMO open-loop plots for the conventional case and the directional notch filtering case are compared around the resonance frequencies of

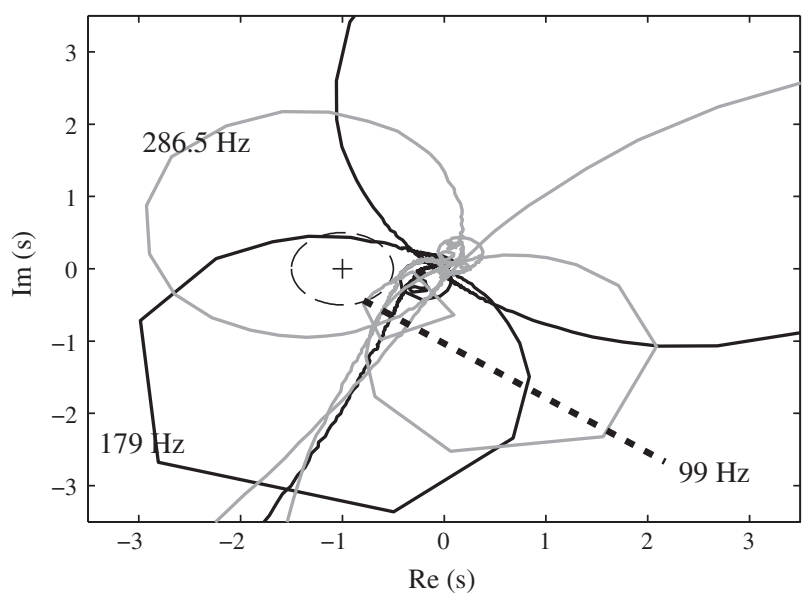

Fig. 8. Characteristic loci of the open loop with lead-lag and low-pass, without notches.

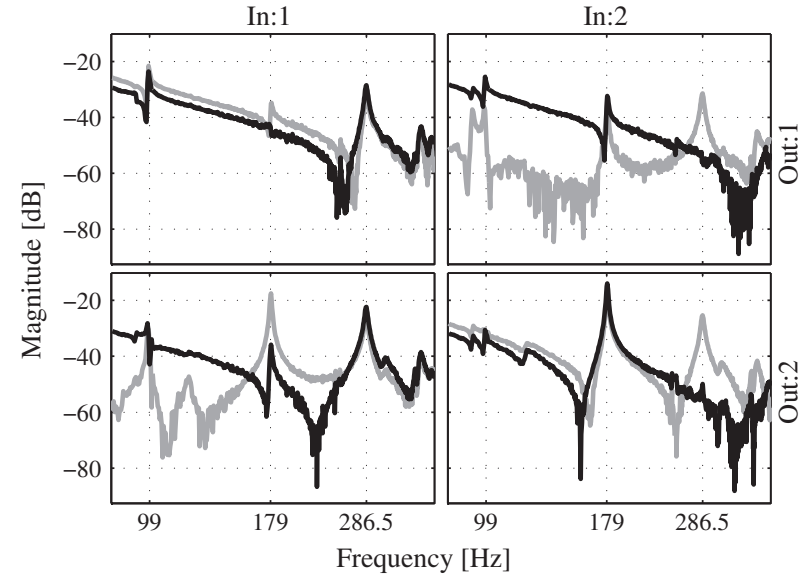

Fig. 9. The system $\mathbf{H}(j \omega) \mathbf{T}_{\text {in,i }}$ (black) does not show the mode at $286.5 \mathrm{~Hz}$ in the second column. The original plant $\mathbf{H}(j \omega)$ is shown in grey.

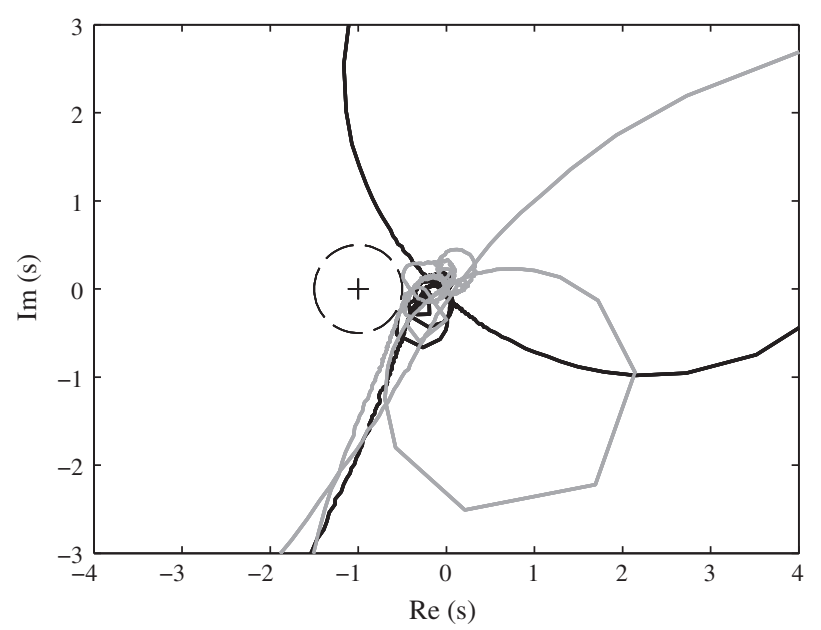

Fig. 10. Characteristic loci of the open loop with lead-lag and low-pass, with directional notches.

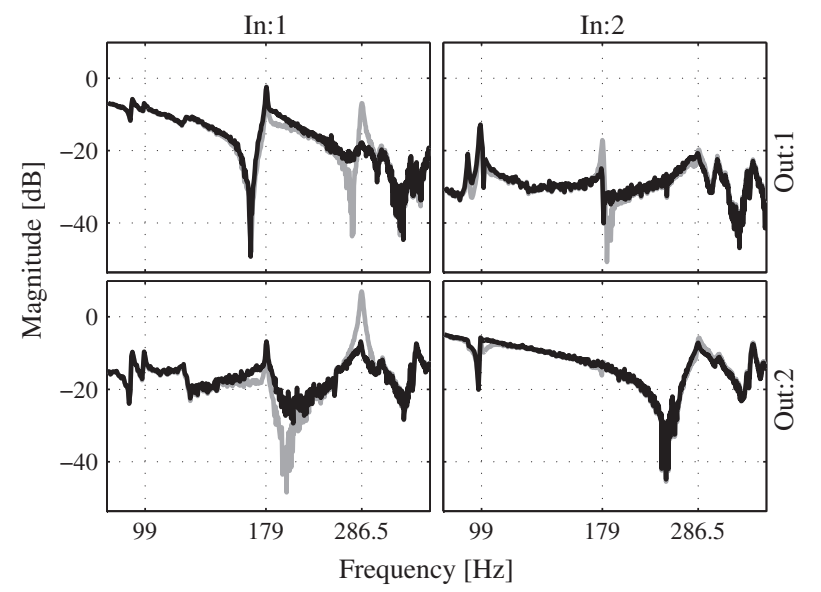

Fig. 11. Open loop for conventional decentralized control (grey) and directional notch filtering approach (black).

the modes, see Fig. 11. In the figure, it can be observed that the resonance peaks in the off-diagonal terms are suppressed much better with the directional notches compared to the conventionally tuned notches. 


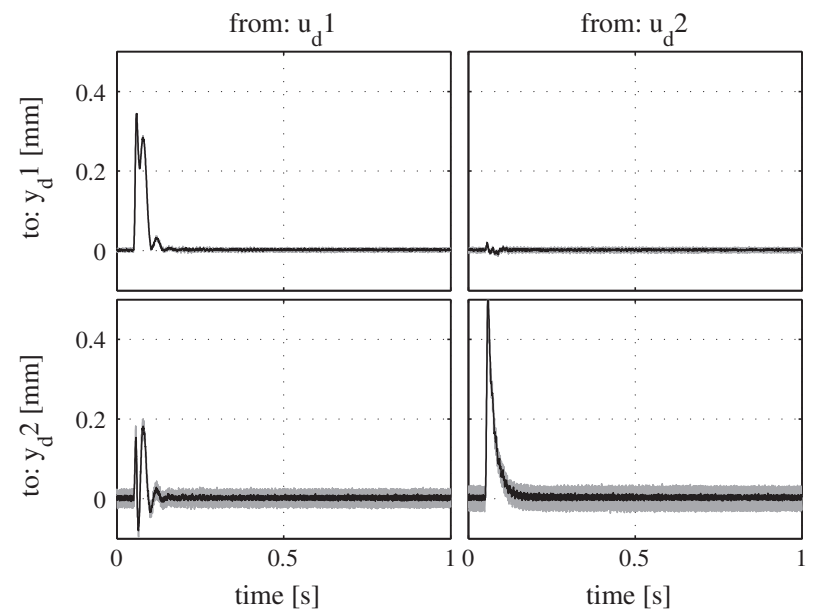

Fig. 12. Impulse response for conventional decentralized control (grey) and directional notch filtering approach (black).

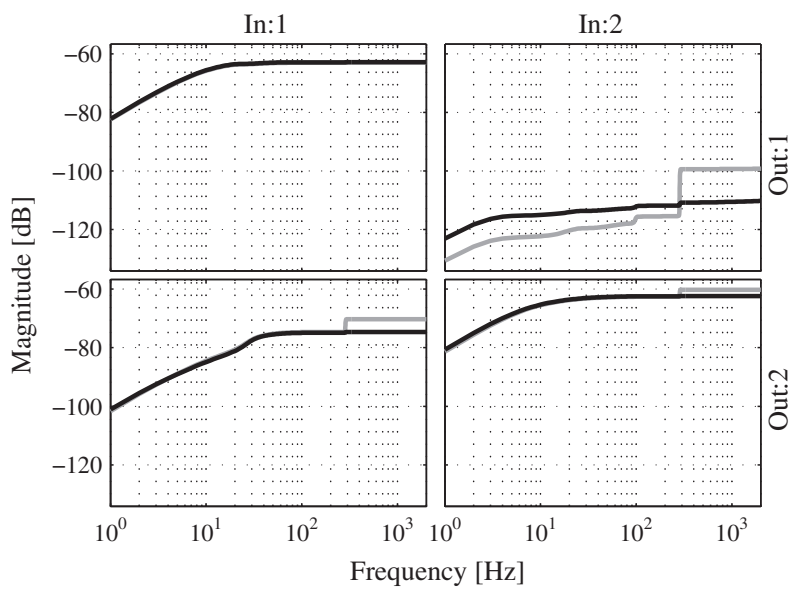

Fig. 13. Cumulative power spectral density plot for conventional decentralized control (grey) and directional notch filtering approach (black).

\subsection{Time domain results}

Time domain results have been obtained from the beam setup for both the conventional loop-shaping controller and the controller with directional notches. Measured MIMO impulse responses are displayed in Fig. 12. Although both controllers are stable, the conventional controller design clearly shows a more oscillatory behaviour. The amplitude of the oscillations is a factor 2-3 larger. The cumulative power spectral density plot of Fig. 13 reveals that this is mainly induced by the resonant mode at $286.5 \mathrm{~Hz}$ that is visible in the response of the conventionally controlled system. This could have been expected as the characteristic loci for the conventional controller lie too close to the Nyquist stability point, recall Fig. 7.

\section{Conclusions}

Directional notch filters provide a new way of loop-shaping notch filters for MIMO motion systems with flexible structures. The mode shapes of the target resonant modes can be used to compute directional notch filters that operate in the correct direction in the MIMO system. The mode shapes of the system are obtained in a data-based way from the measured frequency responses of the system. Only one SISO notch filter is required per mode to suppress a resonance in the MIMO system. Hence, the new approach reduces the order of the controller compared to conventional multiloop
SISO control. The method allows for tuning of the directional notch filters in view of MIMO stability, thereby accounting for the coupling in the system.

Experiments on a prototype lightweight motion system show the feasibility of the approach in practice. Three modes of the beam setup that affect the stability of the closed-loop system are suppressed successfully using directional notches. Compared to conventional multiloop SISO loop-shaping, the results show that suppression of the flexible modes is improved when directional notch filters are applied. This is because directional notch filters account for the directionality of the resonances. Conventional SISO loop-shaping notch filters are not suitable for this, as the system is not decoupled at the frequencies of the resonances.

\section{References}

[1] Butler H. Position control in lithographic equipment. IEEE Control Syst Mag 2011;31(5):28-47.

[2] van de Wal M, van Baars G, Sperling F, Bosgra O. Multivariable feedback control design for high-precision wafer stage motion. Control Eng Pract 2002;10(7):739-55.

[3] Steinbuch M, Norg M. Advanced motion control: an industrial perspective. Eur. J. Control 1998;4:278-93.

[4] Inman DJ. Modal decoupling conditions for distributed control of flexible structures. J Guidance, Control, Dyn 1984;7(6):750-2.

[5] Schneiders M, van de Molengraft M, Steinbuch M. Benefits of over-actuation in motion systems. In: Proc American control conf, Boston, Massachusetts, USA; 2004. p. 505-10.

[6] Friswell M. On the design of modal actuators and sensors. I Sound Vib 2001;241(3):361-72.

[7] Anthonis J, Ramon H. SVD hinf controller design for an active horizontal spray boom suspension. In: Proc 7th Mediterranean conf control and automation, Haifa, Israel; 1999. p. 90-102.

[8] Vaes D. Optimal static decoupling for multivariable control design. Ph.D. thesis, Katholieke Universiteit Leuven; (2005).

[9] Chughtai S, Nobakhti A. Wang H. A systematic approach to the design of robust diagonal dominance based MIMO controllers. In: Proc IEEE conf decision and control, Seville, Spain; 2005. p. 6875-80.

[10] Meirovitch L, Baruh H. On the problem of observation spillover in self-adjoint distributed-parameter systems. J Optim Theory Appl 1983;39(2):269-91.

[11] Balas M. Feedback control of flexible systems. IEEE Trans Autom Control 1978;23(4):673-9.

[12] Inman D. Active modal control for smart structures. Philos Trans R Soc Lond, Ser A 2001;359(1778):208-19.

[13] Cazzulani G, Resta F, Ripamonti F. An application of the IMSC on a non-linear flexible structure: numerical analysis and experimental validation. In: Proc world congress on engineering, vol. III, London, UK; 2011. p. 2221-6.

[14] Resta F, Ripamonti F, Cazzulani G, Ferrari M. Independent modal control for nonlinear flexible structures: an experimental test rig. J Sound Vib 2010:329(8):961-72.

[15] van de Wal M, van Baars G, Sperling F, Bosgra O. Experimentally validated multivariable mu feedback controller design for a high-precision wafer stage. In: Proc IEEE conf decision and control, Orlando, FL, USA; 2001. p. 1583-8.

[16] Gawronski W. Advanced structural dynamics and active control of structures. Springer Verlag; 2004.

[17] Preumont A. Vibration control of active structures: an introduction. 3rd ed. Berlin Heidelberg: Springer Verlag; 2011.

[18] Skogestad S, Postlethwaite I. Multivariable feedback control: analysis and design. John Wiley \& Sons; 2005.

[19] Boerlage M, Middleton RH, Steinbuch M, Jager AGD. Rejection of fixed direction disturbances in multivariable electromechanical motion systems. Mechatronics 2010;20(1):45-52.

[20] Hjalmarsson H, Birkeland T. Iterative feedback tuning of linear time-invariant MIMO systems. In: Proc 37th IEEE conference on decision and control, Tampa, Florida, USA, December 1998. p. 3893-8.

[21] Hara S, Yamamoto Y, Omata T, Nakano M. Repetitive control systema newtype servo system. IEEE Trans Autom Control AC-33 1988:659-88.

[22] Bien Z, Xu JX. Iterative learning control. Analysis, design, integration and applications. Kluwer Academic Publishers; 1998.

[23] Levin J. Adaptive notch filter using real-time parameter estimation. IEEE Trans Control Syst Technol 2011;19(3):673-81.

[24] Hovd M, Skogestad S. Sequential design of decentralized controllers. Automatica 1994;30(10):1601-7.

[25] Mayne DQ. The design of linear multivariable systems. Automatica 1973:9(2):201-7.

[26] Maciejowski J. Multivariable feedback design. Addison-Wesley; 1989.

[27] Reynders E. System identification methods for (operational) modal analysis: review and comparison. Arch Comput Methods Eng 2012;19(1):51-124.

[28] Brown DL, Allemang RJ, Phillips AW. Autonomous modal parameter estimation: application examples. Proc Soc Exp Mech Ser 6, vol. 3. New York (NY, USA): Springer; 2011. p. 403-28. 